\title{
Unresectable Undifferentiated Pleomorphic Sarcoma
}

National Cancer Institute

\section{Source}

National Cancer Institute. Unresectable Undifferentiated Pleomorphic Sarcoma. NCI

Thesaurus. Code C150610.

Undifferentiated pleomorphic sarcoma that is not amenable to surgical resection. 